\title{
ON A THEOREM OF FEJER AND RIESZ
}

\author{
F. R. KEOGH
}

1. In what follows we suppose that $r>1$ and that $A_{r}$ is a constant, depending only on $r$, the value of which is not usually the same at each occurrence. Let $U(\theta)$ denote a real function measurable over $(-\pi, \pi)$, let

$$
P(\rho, \theta)=\left(1-\rho^{2}\right)\left[(1-\rho)^{2}+4 \rho \sin ^{2} \frac{1}{2} \theta\right]^{-1}, \quad 0 \leqq \rho<1,
$$

and let

$$
u(\rho)=\frac{1}{2 \pi} \int_{-\pi}^{\pi} P(\rho, \theta) U(\theta) d \theta .
$$

$P(\rho, \theta)$ is the Poisson kernel and $u(\rho) \equiv u(\rho, 0)$ is the value at the point $(\rho, 0)$ of a function $u(\rho, \theta)$ in polar coordinates which is harmonic inside the unit disc and has boundary value $U(\theta)$ on the unit circle.

We begin by giving a new 'real variable' proof of the following theorem of Fejér-Riesz type. This is similar to a proof given by du Plessis [3] but it differs in a way which leads to a new analogue in three dimensions. Before du Plessis' paper appeared, the only proof available was of a 'complex variable' nature and based on the FejérRiesz inequality $\int_{0}^{1}|f(r)| d r \leqq \frac{1}{2} \int_{-\pi}^{\pi}\left|f\left(e^{i \theta}\right)\right| d \theta[1]$.

Theorem 1.

$$
\int_{0}^{1}|u(\rho)|^{r} d \rho \leqq A_{r} \int_{-\pi}^{\pi}|U(\theta)| r d \theta
$$

The proof is based on the use of an inequality theorem (see, for example, [2, p. 229, Theorem 319]) which we state as a lemma.

Lemma. If $f(x)$ is nonnegative, $K(x, y)$ nonnegative and homogeneous of degree -1 and $\int_{0}^{\infty} K(x, 1) x^{-1 / r} d x=k$, then

$$
\int_{0}^{\infty}\left(\int_{0}^{\infty} K(x, y) f(x) d x\right)^{r} d y \leqq k^{r} \int_{0}^{\infty}(f(x))^{r} d x .
$$

Proof of Theorem 1 . For $0 \leqq \rho<1$, since $P(\rho, \theta)>0$, we have

$$
|u(\rho)| \leqq \frac{1}{2 \pi} \int_{-\pi}^{\pi} P(\rho, \theta)|U(\theta)| d \theta
$$

Received by the editors February 13, 1967. 
so it is enough to prove the theorem under the assumption that $U(\theta) \geqq 0$. We may further assume that $U(\theta)$ is an even function of $\theta$ and prove (what is then equivalent to (1)) that

$$
\int_{0}^{1}\left(\frac{1}{\pi} \int_{0}^{\pi} P(\rho, \theta) U(\theta) d \theta\right)^{r} d \rho \leqq A_{r} \int_{0}^{\pi}(U(\theta))^{r} d \theta,
$$

for on replacing $U(\theta)$ in (3) by the even function $U(\theta)+U(-\theta)$, and using the Hölder inequality $(a+b)^{r} \leqq 2^{r-1}\left(a^{r}+b^{r}\right)$ for $a \geqq 0, b \geqq 0$, we obtain

$$
\begin{aligned}
\int_{0}^{1}|u(\rho)| r d \theta & =\int_{0}^{1}\left[\frac{1}{2 \pi} \int_{0}^{\pi} P(\rho, \theta)(U(\theta)+U(-\theta)) d \theta\right]^{r} d \rho \\
& \leqq A_{r} \int_{0}^{\pi}(U(\theta)+U(-\theta))^{r} d \rho \\
& \leqq A_{r} \int_{0}^{\pi} 2^{r-1}\left[(U(\theta))^{r}+(U(-\theta))^{r}\right] d \theta \\
& =A_{r} \int_{-\pi}^{\pi}(U(\theta))^{r} d \theta .
\end{aligned}
$$

Dividing the range of integration with respect to $\rho$ in (3) into the two intervals $\left(0, \frac{1}{2}\right),\left(\frac{1}{2}, 1\right)$, we first consider integration over $\left(0, \frac{1}{2}\right)$. Since $P(\rho, \theta) \leqq(1+\rho) /(1-\rho)$ we have, using Hölder's inequality,

$$
\begin{aligned}
\int_{0}^{1 / 2}\left(\frac{1}{\pi} \int_{0}^{\pi} P(\rho, \theta) U(\theta) d \theta\right)^{r} d \rho & \leqq \int_{0}^{1 / 2} \pi^{-r}\left(\frac{1+\rho}{1-\rho}\right)^{r}\left(\int_{0}^{\pi} U(\theta) d \theta\right)^{r} d \rho \\
& =A_{r}\left(\int_{0}^{\pi} U(\theta) d \theta\right)^{r} \\
& \leqq A_{r} \int_{0}^{\pi}(U(\theta))^{r} d \theta .
\end{aligned}
$$

Next, defining

$$
P_{1}(\rho, \theta)=\frac{2(1-\rho)}{\left((1-\rho)^{2}+\theta^{2}\right)},
$$

for the interval $\left(\frac{1}{2}, 1\right)$, since $\frac{1}{2} \theta \geqq \theta / \pi$ over $(0, \pi)$, we have

$$
P(\rho, \theta) \leqq \frac{2(1-\rho)}{(1-\rho)^{2}+2 \theta^{2} / \pi^{2}}=\frac{1}{2} \pi^{2} \frac{2(1-\rho)}{\frac{1}{2} \pi^{2}(1-\rho)^{2}+\theta^{2}}<\frac{1}{2} \pi^{2} P_{1}(\rho, \theta),
$$

and so 


$$
\begin{aligned}
& \int_{1 / 2}^{1}\left(\frac{1}{\pi} \int_{0}^{\pi} P(\rho, \theta) U(\theta) d \theta\right)^{r} d \rho \\
& \leqq A_{r} \int_{1 / 2}^{1}\left(\frac{1}{\pi} \int_{0}^{\pi} P_{1}(\rho, \theta) U(\theta) d \theta\right)^{r} d \rho
\end{aligned}
$$

With $f(x)=U(x)$ for $0 \leqq x \leqq \pi, f(x)=0$ for $x>\pi$, and

$$
K(x, y)=(2 / \pi) y /\left(x^{2}+y^{2}\right),
$$

the functions $f(x), K(x, y)$ satisfy the conditions of the lemma (with $\left.k=\operatorname{cosec} \frac{1}{2} \pi(1-1 / r)\right)$. On replacing $x$ by $\theta, y$ by $1-\rho,(2)$ and (5) give

$$
\int_{1 / 2}^{1}\left(\frac{1}{\pi} \int_{0}^{\pi} P_{1}(\rho, \theta) U(\theta) d \theta\right)^{r} d \rho \leqq \int_{-\infty}^{1} \leqq A_{r} \int_{0}^{\pi}(U(\theta))^{r} d \theta
$$

and finally (4) and combination of (6) and (7) give the inequality (3). This completes the proof of the theorem.

2. When analogues of Theorem 1 for functions harmonic in the unit sphere are considered there are two possibilities. Let $(\rho, \theta, \phi)$ denote spherical polar coordinates, $U(\theta, \phi)$ a real function measurable for $0 \leqq \theta \leqq \pi,-\pi \leqq \phi \leqq \pi$, and let

$$
\begin{aligned}
Q(\rho, \theta) & =\left(1-\rho^{2}\right)\left[(1-\rho)^{2}+4 \rho \sin ^{2} \frac{1}{2} \theta\right]^{-3 / 2} \sin \theta, \\
u(\rho) & =\int_{0}^{\pi}\left(\int_{-\pi}^{\pi} U(\theta, \phi) d \phi\right) Q(\rho, \theta) d \theta .
\end{aligned}
$$

Here $Q(\rho, \theta)$ is the three-dimensional Poisson kernel and $u(\rho)$ $\equiv u(\rho, 0,0)$ is the value at the point $(\rho, 0,0)$ of a function $u(\rho, \theta, \phi)$ harmonic inside the unit sphere and with boundary values $U(\theta, \phi)$ on the surface. The possibilities are

$$
\int_{0}^{1}\left(\int_{0}^{\rho}|u(r)| r d t\right) d \rho \leqq A_{r} \int_{0}^{\pi} \int_{-\pi}^{\pi}|U(\theta, \phi)|^{r} \sin \theta d \phi d \theta .
$$

and

$$
\int_{0}^{1}|u(\rho, \theta, \phi)|{ }^{r} \rho d \phi d \theta \leqq A_{r} \int_{0}^{\pi} \int_{-\pi}^{\pi}|U(\theta, \phi)| r \sin \theta d \phi d \theta .
$$

In both inequalities the right-hand side is the integral of $|U(\theta, \phi)|^{r}$ over the surface of the unit sphere. In the first inequality, the lefthand side consists of two integrations of $u(\rho, \theta, \phi)$ along a radius, in the second inequality the left-hand side is the integral of $u(\rho, \theta, \phi)$ over a diametral plane. Both of these analogues are, in fact, valid and 
they are particular cases of a general theorem of du Plessis [3] concerning functions in $n$ dimensions. du Plessis' proof of this general theorem is indirect and depends on half-space analogues of Theorem 1 [4]. In this note we give a direct proof of a stronger version of (8) which does not seem to be deducible using du Plessis' argument.

THEOREM 2.

$$
\int_{0}^{1}(1-\rho)|u(\rho)|^{r} d \rho \leqq A_{r} \int_{0}^{\pi}\left(\int_{-\pi}^{\pi}|U(\theta, \phi)| d \phi\right)^{r} \sin \theta d \theta .
$$

The left-hand side here is identical to the left-hand side of (8) and, by Hölder's inequality,

$$
\int_{0}^{\pi}\left(\int_{-\pi}^{\pi}|U(\theta, \phi)| d \phi\right)^{r} \sin \theta d \theta \leqq A_{r} \int_{0}^{\pi} \int_{-\pi}^{\pi}|U(\theta, \phi)|^{r} \sin \theta d \phi d \theta,
$$

so that (9) is a stronger inequality than (8).

Proof. Arguing as before, it is enough to prove the theorem under the assumption that $U(\theta, \phi) \geqq 0$.

We divide the range of integration with respect to $\rho$ as before, and first consider integration over $\left(0, \frac{1}{2}\right)$. Since

$$
Q(\rho, \theta) \leqq(1+\rho)(1-\rho)^{-2} \sin \theta
$$

we have, using Hölder's inequality,

$$
\begin{aligned}
\int_{0}^{1 / 2}(1 & -\rho)(u(\rho))^{r} d \rho \\
= & \int_{0}^{1 / 2}(1-\rho)\left[\frac{1}{4 \pi} \int_{0}^{\pi}\left(\int_{-\pi}^{\pi} U(\theta, \phi) d \phi\right) Q(\rho, \theta) d \theta\right]^{r} d \rho \\
\leqq & \int_{0}^{1 / 2}(1-\rho)(4 \pi)^{-r} \frac{(1+\rho)^{r}}{(1-\rho)^{2 r}} \\
& \cdot\left[\int_{0}^{\pi}\left(\int_{-\pi}^{\pi} U(\theta, \phi) d \phi\right) \sin \theta d \theta\right]^{r} d \rho \\
= & A_{r}\left[\int_{0}^{\pi}\left(\int_{-\pi}^{\pi} U(\theta, \phi) d \phi\right) \sin \theta d \theta\right]^{r} \\
\leqq & A_{r} \int_{0}^{\pi}\left(\int_{-\pi}^{\pi} U(\theta, \phi) d \phi\right)^{r} \sin r \theta d \theta \\
\leqq & A_{r} \int_{0}^{\pi}\left(\int_{-\pi}^{\pi} U(\theta, \phi) d \phi\right)^{r} \sin \theta d \theta .
\end{aligned}
$$


Next, defining

$$
R(\rho, \theta)=2(1-\rho)^{1+1 / r} \theta^{1-1 / r}\left[(1-\rho)^{2}+\theta^{2}\right]^{-3 / 2}
$$

for the interval $\left(\frac{1}{2}, 1\right)$, since $\sin \theta \leqq \theta$ and $\sin \frac{1}{2} \theta \geqq \theta / \pi$ over $(0, \pi)$, we have

$$
\begin{aligned}
Q(\rho, \theta) & \leqq 2(1-\rho)\left[(1-\rho)^{2}+2 \theta^{2} / \pi^{2}\right]^{-3 / 2} \sin \theta \\
& =2^{-1 / 2} \pi^{3}(1-\rho)\left[\frac{1}{2} \pi^{2}(1-\rho)^{2}+\theta^{2}\right]^{-3 / 2} \sin \theta \\
& \leqq 2^{-1 / 2} \pi^{3}(1-\rho)\left[(1-\rho)^{2}+\theta^{2}\right]^{-3 / 2} \sin \theta \\
& \leqq 2^{-3 / 2} \pi^{3}(1-\rho)^{-1 / r} \sin ^{1 / r} \theta R(\rho, \theta),
\end{aligned}
$$

and so

$$
\begin{aligned}
\int_{1 / 2}^{1}(1 & -\rho)(u(\rho))^{r} d \rho \\
& =\int_{1 / 2}^{1}(1-\rho)\left[\frac{1}{4 \pi} \int_{0}^{\pi}\left(\int_{-\pi}^{\pi} U(\theta, \phi) d \phi\right) Q(\rho, \theta) d \theta\right]^{r} d \rho \\
& \leqq A_{r} \int_{1 / 2}^{1}\left[\frac{1}{4 \pi} \int_{0}^{\pi} R(\rho, \theta)\left(\int_{-\pi}^{\pi} U(\theta, \phi) d \phi\right) \sin ^{1 / r} \theta\right]^{r} d 0
\end{aligned}
$$

Defining $f(x)=\sin ^{1 / r} x \int_{-\pi}^{\pi} U(x, \phi) d \phi$ for $0 \leqq x \leqq \pi, f(x)=0$ for $x>\pi$, and

$$
K(x, y)=(2 \pi)^{-1} x^{1-1 / r} y^{1+1 / r}\left(x^{2}+y^{2}\right)^{-3 / 2},
$$

the functions $f(x), K(x, y)$ satisfy the conditions of the lemma (with $\left.k=\frac{1}{2} \pi^{-3 / 2} \Gamma(1-1 / r) \Gamma(1 / 2+1 / r)\right)$. On replacing $x$ by $\theta, y$ by $1-\rho$, (2) and (11) give

$$
\begin{aligned}
& \int_{1 / 2}^{1}\left[\frac{1}{4 \pi} \int_{0}^{\pi} R(\rho, \theta)\left(\int_{-\pi}^{\pi} U(\theta, \phi) d \phi\right) \sin ^{1 / r} \theta\right]^{r} d \rho \\
& \leqq A_{r} \int_{0}^{\pi}\left(\int_{-\pi}^{\pi} U(\theta, \phi) d \theta\right)^{r} \sin \theta d \theta .
\end{aligned}
$$

Combination of (12) and (13) now gives

$$
\int_{1 / 2}^{1}(1-\rho)(u(\rho))^{r} d \rho \leqq A_{r} \int_{0}^{\pi}\left(\int_{-\pi}^{\pi} U(\theta, \phi) d \phi\right)^{r} \sin \theta d \theta
$$

and addition of (10) and (14) yields the desired inequality (9). 


\section{REFERENCES}

1. L. Fejér and M. Riesz, Über einige funktiontheoretische Ungleichungen, Math. Z. 11 (1921), 305-314.

2. G. H. Hardy, J. E. Littlewood and G. Polya, Inequalities, Cambridge Univ. Press, Cambridge, 1934.

3. N. du Plessis, Spherical Fejêr-Riesz theorems, J. London Math. Soc. 31 (1956), 386-391.

4. - Half-space analogues of the Fejer-Riesz theorem, J. London Math. Soc. 30 (1955), 296-301.

UNIVERSITY OF KENTUCKY 